\title{
Impacto del Ciberbullying en la Depresión de Estudiantes Universitarios del Distrito de Lima
}

\section{Cyberbullying Impact on Depression of University Students from Lima District}

\author{
Betzabeth Rosella Valle Gonzales ${ }^{1}$ \\ Universidad Cesar Vallejo, Perú \\ https://orcid.org/0000-0001-5775-7838
}

Recibido: $17-08-2021$

Aceptado: 22-12-2021

\section{Cita Recomendada}

Valle, B. (2021). Impacto del ciberbullying en la depresión de estudiantes universitarios del distrito de Lima. Hamut'ay, 8 (3), pag. 15-31, http://dx.doi.org/10.21503/hamu.v8i3.2325

\section{Resumen}

La investigación presentada a continuación planteó como propósito conocer el impacto del ciberbullying sobre la construcción y desarrollo de la depresión en población universitaria de Lima. Se usaron análisis de correlación y regresión múltiple. El sector de la población considerado para dicho estudio, se constituyó por 150 universitarios del distrito de Lima cuyas edades oscilaron entre los 16 y los 19 años. Se emplearon escalas de tipo Likert para la recopilación de la información; así mismo, a través del análisis de los datos, se determinó la incidencia del ciberbullying, sobre la depresión en los estudiantes encuestados $(\mathrm{F}(2,114)=22,748$, $\mathrm{p}<.001 ; \mathrm{R} 2$ ajustado $=0.272)$. El análisis de correlación demostró la relación significativa y positiva de la Cibervictimización verbal - escrita $(\rho=0.45, \mathrm{p}=.02)$ con la depresión y el análisis de regresión evidenció que dicho constructo se relaciona con el $23 \%$ de la varianza de la depresión $(B=0.771, t=3.231, \mathrm{p}<.01)$ y con el $8 \%$ de la exclusión online $(\mathrm{B}=1.956, \mathrm{t}=2.756, \mathrm{p}<.01)$. Respecto a los otros tipos de ciberbullying: cibervictimización visual, exclusión y suplantación, pese que el análisis de correlación presentó coeficientes significativos que oscilaron entre $\rho=0.37$ y el $\rho=0.45$, el análisis de regresión múltiple no los incluyó dentro del modelo como variables predictoras.

Palabras Clave: ciberbullying, depresión, adolescentes

1. Docente de la Universidad César Vallejo y docente de la Universidad Tecnológica del Perú. Maestra en Psicología educativa. Doctorando del VI ciclo. betzabeth.vg@gmail.com 


\begin{abstract}
The present research aims to establish the impact of cyberbullying on depression in university students in the district of Lima.

Descriptive, correlation and multiple regression analyzes were used. The sample consisted of 150 university students from the Lima district aged between 16 and 19 years. The instruments used were questionnaires, Likert scale type, and correlation and regression analyze were performed. The incidence of cyberbullying on depression in the surveyed students was determined $(\mathrm{F}(2,114)=22.748, \mathrm{p}<.001$; adjusted $\mathrm{R} 2=0.272)$. The correlation analysis demonstrated the significant and positive relationship of verbal-written cybervictimization $(\rho=0.45, p=.02)$ with depression and the regression analysis showed that the verbal-written cybervictimization dimension is related to $23 \%$ of the variance of depression $(B=0.771, t=3.231, p<.01)$ and online exclusion with $8 \%(\mathrm{~B}=1.956, \mathrm{t}=2.756, \mathrm{p}<.01)$. Regarding the other types of cyberbullying: visual cybervictimization, exclusion and impersonation in depression, although the correlation analysis between the variables presented significant coefficients that ranged between $\rho=0.37$ and $\rho=0.45$ ), the multiple regression analysis did not include them within the model as predictor variables.
\end{abstract}

Key Words: cyberbullying, depression, adolescents

\section{Introducción}

Es evidente que en la actualidad las Tecnologías de la Información y la Comunicación (TIC) desempeñan un rol de suma importancia en el quehacer diario de los adolescentes, al igual que las redes sociales (Renés-Arellano et al., 2021), constituyen herramientas básicas para el desarrollo de actividades de entretenimiento, académicas, interacción social, ocio y demás (Matamala, 2016). Sin embargo, su uso indebido a la larga puede desencadenar una serie de conductas violentas, lo que se denomina ciber violencia o violencia cibernética, en referencia a todo comportamiento que vulnera el bienestar psicológico y emocional de quien lo padece, ya sea por medio de prácticas como el hostigamiento, el acoso cibernético o la violencia verbal, entre otras manifestaciones; cada una de ellas ejecutadas con ayuda y bajo el uso de las TIC (Stopcyberbullying, s.f.).

Los efectos de mayor gravedad provocados por este fenómeno y la percepción del daño, encuentran respuesta en las características de personalidad de cada individuo, los rasgos de comportamiento más representativos de la víctima, el modo de acoso y el apoyo que se le brinda (Hoff y Mitchell, 2009; Garaigordobil, 2010). En esa línea, la práctica del ciberbullying alcanza tal repercusión sobre las víctimas, que es capaz de desencadenar una serie de efectos negativos a nivel psicológico sobre ellas, siendo, algunas de estas, el inicio y desarrollo de alteraciones a nivel alimenticio, del sueño, del aislamiento social y sin restar importancia a las anteriores, hay una que en especial ha hecho que las cifras vayan en aumento como la depresión (Kim, 2018).

La depresión en población adolescente conforma un fenómeno de salud mental de considerable preocupación, caracterizado por el constante sentimiento de tristeza y pérdida de interés frente al ejercicio de las actividades cotidianas; del mismo modo repercute en el sentir, actuar y pensar del adolescente, pudiendo dar paso a complicaciones de tipo físico, emocionales o funcionales (OMS, 2017; Alvites-Huamaní, 2020). Aunque el trastorno depresivo no considera etapas de vida específicas para su manifestación, el fácil acceso que en especial pueden tener los adolescentes a Internet, puede facilitar muchos aspectos de su vida, pero también puede ser una fuente para realizar conductas negativas como el ciberbullying (Garaigordobil, 2015).

Dada la importancia que tienen estos fenómenos en las áreas educativas, especialmente en la educación universitaria, y teniendo en cuenta que durante este periodo de vida los jóvenes 
estudiantes presentan cambios emocionales y comportamentales, la ejecución de acciones contra el ciberbullying que puedan fomentar y contribuir de manera significativa en el desarrollo de la depresión en las víctimas que la experimentan, se torna indispensable. Por lo antes mencionado la presente investigación propone como objetivo, conocer el impacto del ciberbullying y sus diferentes manifestaciones sobre la depresión en estudiantes universitarios de la ciudad de Lima. Y se propone como hipótesis general que los diferentes tipos de ciberbullying impactan de manera positiva y significativa en la variable depresión de dichos estudiantes.

\section{Ciberbullying: conceptos, características y} consecuencias

Smith (2006) conceptualiza el Ciberbullying como el conjunto de conductas agresivas intencionadas y deliberadas puestas en práctica sobre un grupo específico de individuos, teniendo como principal herramienta las redes sociales, a través de las cuales violentan a aquellos que carecen de capacidad para protegerse por sí mismas.

Si bien el ciberbullying comparte una serie de particularidades con el bullying o acoso escolar, presenta algunas singularidades como: 1) Gran alcance y amplio número de espectadores virtuales por la publicación de cualquier tipo de contenido digital, cuyo objetivo es denigrar y dañar la reputación de una persona en específico, dado que los receptores que albergan las redes sociales superan considerablemente en número a los hallados en un entorno físico, lo que a su vez intensifica los efectos negativos sobre la víctima (Walrave, M., \& Walrave, H., 2009).

2) La posibilidad de crear cuentas falsas y mínimas restricciones que ofrecen las redes sociales, crean la sensación de impunidad para acosadores virtuales, quienes valiéndose del anonimato e invisibilidad ejercen comportamientos violentos sobre las víctimas, quienes reciben dichos ataques con angustia y desconcierto, y sobre las cuales no se generan sentimientos de culpa y empatía, como es el caso de los acosadores presenciales (Willard, 2006). 3) El funcionamiento del ciberbullying es imparable, de 24 horas al día, y fácil acceso dadas las posibilidades que brinda el Internet, lo que permite al acosador cibernético asediar con mayor continuidad a su víctima, sobre la cual se genera un tipo de agravio permanente de mayor intensidad, debido a que el contenido expuesto permanece de manera indefinida en las plataformas virtuales, siendo posible su eliminación únicamente con la intervención de las autoridades pertinentes ( $\mathrm{Li}, 2008)$. 4) $\mathrm{La}$ velocidad y simpleza con la que las TIC permiten la difusión de información, posibilita al acosador cumplir con su objetivo de perjudicar mediante la propagación de contenido digital, a diferencia del asedio tradicional, en donde el atacante hace uso del contacto físico, por medio de golpes, insultos, entre otros, para intimidar a su víctima (Smith, 2006). 5) La poca visibilización del agresor que ofrece los medios digitales, imposibilitan la identificación de la imagen del agresor, quienes pueden mostrar un perfil impecable, mientras que en el acoso escolar por lo general el agresor se caracteriza por conformar una imagen social negativa, en donde la indisciplina y conducta violenta conforman algunas de sus principales particularidades.

Giménez (2015) enfatiza en el desarrollo de tres roles participantes en el fenómeno del ciberbullying, siendo estos: el rol de ciberagresor, la cibervíctima y ciberespectador. El rol del ciberagresor, hace referencia al individuo que hace uso de las TIC para a través de dicho medio lograr el objetivo de hostigar a su víctima. Asimismo, señala que, entre sus principales características, se destacan la poca empatía, así como el desarrollo deficiente de la habilidad para interactuar con su medio sin la necesidad de delimitar las diferencias de poder. En cuanto a los agresores refiere que se muestran como sujetos ambiciosos, con altos deseos de liderar frente a un determinado grupo, teniendo en su mayoría reacciones impulsivas y por lo general negativas en el proceso de socialización. El rol de las cibervíctimas, describe a aquellos individuos víctimas del asedio que utilizan las plataformas digitales como herramientas de acoso. Domínguez y Portela (2020) y Giménez (2015) 
mencionan que, pese a que cualquier persona es vulnerable a experimentar hostigamiento de este tipo, tienen algunas características particulares como escasa o nula popularidad y aceptación dentro del grupo, sentimientos de soledad, culpa, temperamento por lo general débil, inseguro, bajos niveles de autoestima e inadecuado autoconcepto. $\mathrm{Y}$ el rol de los ciberobservadores, engloba a aquellos espectadores o conocedores de la existencia de casos de violencia por ciberbullying que mantienen una actitud indiferente frente a dicho fenómeno, siendo considerados agresores pasivos, de manera que teniendo la posibilidad de denunciar los hechos a las autoridades competentes, mantienen una posición de neutralidad, lo cual contribuye y colabora con que dicha modalidad de violencia se perpetúe.

Álvarez-García, Dobarro y Núñez (2014) tipifican el ciberbullying en cuatro aspectos, los cuales se describen en la Figura 1.

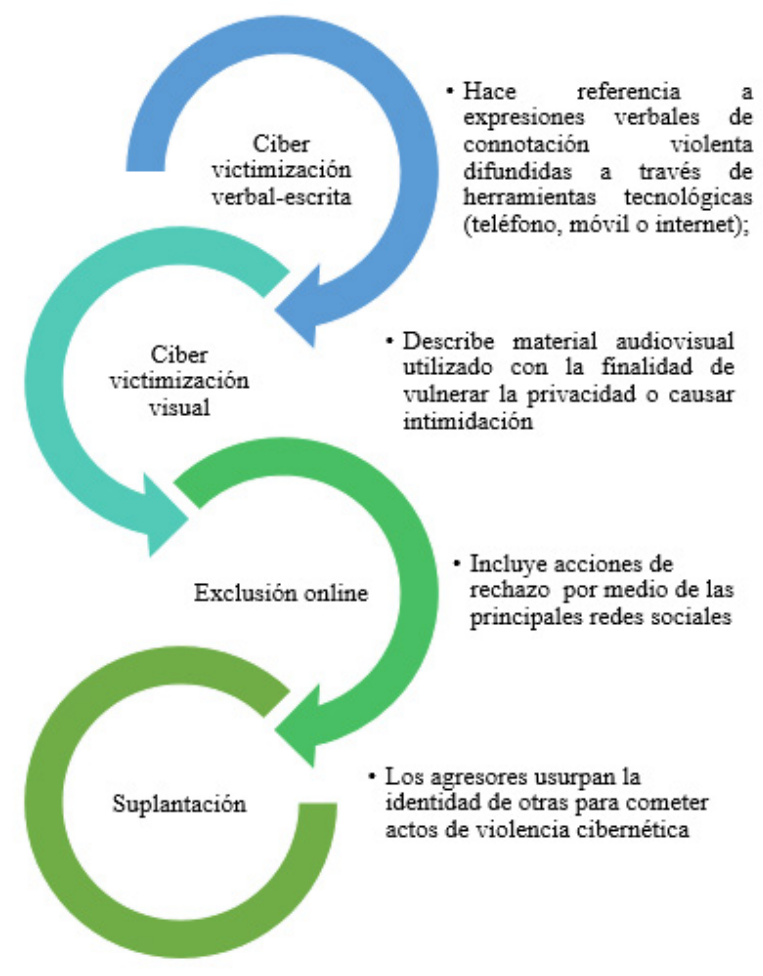

Figura 1. Tipificación del ciberbullying Fuente: Álvarez-García, Dobarro y Núñez (2014)
Por su parte Ybarra y Mitchell (2004) refieren que los efectos del acoso cibernético se consideran de mayor magnitud en relación a otras manifestaciones de violencia, porque afectan de manera transversal los espacios de interacción del individuo que lo padece como el entorno familiar, amical o escolar, por lo que surge en él, la sensación de no sentirse seguro en ninguno de estos ambientes. En el ámbito académico algunos de los efectos alcanzados como consecuencia del hostigamiento cibernético, se encuentra el ausentismo o abandono académico, de manera que el estudiante en su intento de huir, evita formar parte del ambiente y contexto en donde se desarrolla la agresión, o el acercamiento con quienes lo cometen, así mismo, es frecuente el uso y abuso de sustancia psicoactivas, alcohol o drogas, afectando considerablemente su salud física y psicológica (Willard, 2006). Otra de las consecuencias se evidencia en las dificultades asociadas al entorno y desarrollo académico, de modo que se ve afectada la capacidad para disfrutar de las actividades inherentes al entorno, dificultades en el aprendizaje, ausencias o deserción escolar, y el inicio de una serie de condiciones psicológicas, como trastorno por estrés post traumático, problemas en la comunicación de lo experimentado a padres, profesores y demás figuras de autoridad (Brewer y Kerslake, 2015). En esa misma línea, el contenido violento que albergan las redes sociales como publicaciones y comentarios con connotación ofensiva (Rumiche y Solís, 2021), pueden llegar a desencadenar en las víctimas cuadros depresivos y otras condiciones psicológicas, en donde la autoestima y en general la salud psicológica de los sujetos se ve gravemente afectada. Del mismo modo, las dinámicas familiares, en donde no se preserva y practica la comunicación asertiva $y$ confianza entre sus miembros se ven deterioradas, Finalmente se han registrado consecuencias irreversibles a causa del hostigamiento o acoso cibernético, frente a situaciones en donde los individuos que lo padecen al encontrarse sin salida ni alternativas de solución optan por atentar contra la propia vida (Girgin, 2019; Hinduja y Patchin, 2010; Tajahuercel, Franco y Juárez, 2018). 


\section{La depresión en los adolescentes}

Beck y Alford (2009) definen a la depresión como un efecto directo proveniente de la distorsión cognitiva y el modo de razonar de la persona que la padece; desencadenando manifestaciones como la pérdida del interés por las actividades cotidianas, déficit en la motivación, actitud pasiva, entre otros rasgos que asume el individuo, propios de la alteración e interacción con la realidad que percibe. Beck (1987), postula, además, que se trata de un trastorno de gran incidencia en pacientes que sufren algún tipo de afección medida, por lo que su tratamiento sugiere intervención primaria, vinculada con una discapacidad funcional de considerable preocupación. Así mismo, destaca que la depresión tiene su origen multicausal, el cual integra factores psicosociales, genéticos y biológicos.

La Organización Mundial de la Salud por su parte, ubican a la depresión dentro del grupo de trastornos mentales más frecuentes, al concentrar manifestaciones como alteraciones en el sueño y apetito, sentimiento constante de tristeza, llanto y abatimiento, ansiedad, pensamientos automáticos que consideran la posibilidad de suicidio (OMS, 2017).

Una de las manifestaciones de la depresión en los adolescentes es la alteración en la forma en la que perciben su entorno, a sí mismos y la idea de cómo los distinguen de los demás. Es habitual que, durante esta etapa de vida, aquellos jóvenes que padecen de depresión posean una perspectiva negativa de lo que sucede a su alrededor, encontrando dificultad en el planteamiento de soluciones, y en el desarrollo de la capacidad para resolver problemas con una mirada positiva de la vida. La adolescencia, además, conforma un periodo en donde surgen una serie de cuestionamientos y desconciertos a los cuales el individuo no siempre encuentra respuesta, lo que intensifica el destemple emocional. Al respecto Craig (2001), describe a los adolescentes como una población altamente vulnerable, en donde el padecer depresión a su vez puede desencadenar comorbilidades ligadas a otros trastornos mentales, entre ellos el trastorno de la conducta alimentaria, ansiedad, abuso de sustancias y conducta suicida.

Beck (1987) precisa que los niños y adolescentes deprimidos, han construido a lo largo de su crecimiento esquemas mentales negativos, influenciados por acontecimientos traumáticos y eventos desfavorables ocurridos en los diferentes ámbitos de desarrollo, tales como el rechazo social, acoso escolar, críticas por parte de las maestras e incluso antecedentes de depresión por parte de las figuras parentales. Dichos esquemas, se activan cuando la persona es expuesta a situaciones que le recuerdan o asocian hechos pasados, lo que, apoyados en distorsiones cognitivas, dan lugar a una percepción errónea de la realidad.

El modelo establecido por Beck (1987), propone tres niveles de depresión; el nivel leve, donde el individuo pierde el interés por las actividades cotidianas desarrolladas en el entorno laboral, personal y social. El nivel medio, describe al individuo como incapaz de controlar todas aquellas cogniciones desafortunadas sobre sí mismo y el contexto en donde se desenvuelve. Finalmente, en el nivel más severo se evidencian síntomas como aislamiento, distraibilidad, sensación de angustia, ansiedad, entre otros.

En la actualidad, la depresión constituye una de las alteraciones mentales con mayor incidencia a nivel mundial (Tabares et al., 2020). La OMS (2021) reportó que la depresión es la principal causa de discapacidad a nivel mundial que contribuye de gran manera a la carga general de morbilidad; además se estima que 280 millones de personas padecen de dicha condición, pues a pesar de la existencia de un tratamiento son mínimos los casos de los que se requiere o se solicita ayuda.

Estudios empíricos sobre la relación entre ciberbullying y depresión

Diversos estudios han evaluado la relación de esas variables a nivel internacional utilizando en su mayoría análisis correlacionales. Dervishi, Lala y Ibrahimi (2019) exploran en Albania la relación 
entre el bullying y los síntomas de depresión en 284 adolescentes en un rango de edades de 13 a 18 años; encontraron que la victimización es un indicador del acoso correlacionada positivamente $\mathrm{y}$ de manera moderada con depresión $(\mathrm{r}=0.3 ; \mathrm{p}<.05)(\mathrm{r}=0,295 ; \mathrm{p}<0.3)$. Por otro lado, Arhin et al. (2019), realizaron un estudio en Ghana cuyo objetivo fue explorar la relación entre la victimización de bullying y la angustia psicológica, siendo considerada una de ellas la depresión, con 198 adolescentes en edades que oscilaban los 11 y 19 años. Se encontró que existe asociación positiva entre la victimización por acoso escolar y la depresión $(\mathrm{r}=0.35, \mathrm{p}<$ .001) así como con el estrés y ansiedad.

Otro estudio que evaluó la relación entre ambas variables fue el de Cañas et al. (2019), que propuso como objetivo analizar los rasgos psicológicos de 1318 adolescentes involucrados en el fenómeno del ciberacoso en cuatro instituciones educativas del nivel secundaria de Andaluza, Aragonesa y Valenciana, con edades entre 11 y 18 años. Las variables consideradas para el análisis fueron autoconcepto, soledad, sintomatología depresiva, estrés percibido, ansiedad social, satisfacción con la vida e inteligencia emocional. El análisis de los resultados, evidenciaron que el grupo cibervíctimas graves, presentaron un puntaje elevado con respecto a las variables estrés, soledad, depresión y ansiedad. Mientras tanto el grupo de ciberacosadores grave presentó puntuaciones altas en las dimensiones estrés, soledad y depresión y valores bajos en las variables autoconcepto y satisfacción con la vida. Hill et al. (2016) estudiaron la relación entre los constructos victimización y perpetración del bullying cibernético y su implicación en el desarrollo de los síntomas depresivos en población adolescente. Respecto a las diferencias halladas en los grupos perpetradores, las víctimas reportaron sintomatología depresiva en niveles muy superiores a las halladas en los agresores, pese a que el valor absoluto de esta diferencia fue pequeño. Por otro lado, la investigación de Ortega-Barón et al. (2016) se enfocaron en conocer las diferencias existentes entre los constructos satisfacción con la vida, sintomatología depresiva y soledad de las víctimas de acoso cibernético considerando como factores diferenciales el tiempo de duración en relación a la experiencia: (no me ha pasado, 1 mes (o menos), entre 3 y 6 meses, y 1 año (o más). Para ello, se trabajó sobre una muestra conformada por 785 adolescentes con edades de entre 12 y 18 años, residentes de la ciudad de Valencia. Los hallazgos reportaron que las víctimas de ciberbullying, independientemente del tiempo que hayan padecido el acoso, presentan sintomatología depresiva, mientras que los que vivenciaron el hostigamiento por un año o más presentan altos niveles respecto al sentimiento de soledad.

En Perú, Arévalo (2020) realizó una investigación sobre el ciberbullying y la depresión a nivel secundario en población estudiantil asistente a escuelas públicas del distrito del Rímac. El sector de la población se conformó por 300 adolescentes cuyas edades comprendieron entre los 13 y 17 años. Los hallazgos determinaron la existencia de relación positiva, media y significativa ( rho $\mathrm{m}=$ $0.39, \mathrm{p}<.00$ ) entre ciberbullying y depresión. Por otro lado, Alvites (2019) realizó una investigación cuya finalidad fue establecer la relación entre las variables ciber victimización verbal y escrita, visual, exclusión online, suplantación y depresión en población estudiantil del nivel secundaria, pertenecientes a una institución pública de mujeres en el departamento de Piura. La muestra estuvo conformada por 363 estudiantes cuyas edades oscilaron entre los 11 y 16 años y los resultados a los que se arribaron comprobaron la existencia de relación entre el ciberbullying y la depresión $(\mathrm{p}=.020)$. Se determinó además que los factores de mayor incidencia sobre la depresión son la cibervictimización verbal y escrita $(\mathrm{p}=$ .010); aunque es mayor la significancia hallada en la relación cibervictimización visual y depresión $(\mathrm{p}=.024)$. Por otro lado, no se halló asociación entre la exclusión online y suplantación.

Sandoval y Caballero (2016) realizaron un estudio cuyo propósito fue determinar la relación entre las variables bullying, ciberbullying $y$ conducta suicida en 289 escolares de secundaria de instituciones educativas de la ciudad de Piura. El análisis multivariado evidenció que en 
mayor porcentaje los individuos que ejercieron conductas suicidas presentaron los siguientes factores predisponentes como ser víctimas de bullying, la variable sociodemográfica sexo, siendo el género femenino el de mayor prevalencia, el nivel de depresión, medido a través del cuestionario de depresión infantil de Kovacs (CDI) y el riesgo suicida evaluada por medio de Escala de Riesgo Suicida (ERS).

\section{Materiales y métodos.}

Participantes

La población considerada para la investigación se conformó por estudiantes universitarios residentes en el Distrito de Lima. La muestra estuvo conformada por 150 participantes (Tabla 1). El tipo de muestreo considerado fue no probabilístico de tipo intencional, de manera que es el investigador quien la escoge a partir de supuestos generales de la población seleccionada para la evaluación (Hernández et al., 2014) y se consideró los criterios de inclusión: 1) alumnos de universidades de la ciudad de Lima de ambos sexos que asisten regularmente a clases. 2) Que decidan voluntariamente formar parte del estudio. Y los siguientes criterios de exclusión: 1) que no asistan regularmente a clases, 2) que no deseen participar en la investigación. 3) que no hayan respondido a todas las preguntas del cuestionario.

Tabla 1.Estadísticas descriptivas delas variables sociodemográficas

\begin{tabular}{ccc}
\hline \multicolumn{2}{l}{ Variable sociodemográfica } \\
\hline Sexo & Número & Porcentaje \\
\hline Femenino & 123 & $81.5 \%$ \\
Masculino & 27 & $17.9 \%$ \\
\hline & Media & Desviación Estándar \\
\hline Edad & 18 & 0.97 \\
\hline
\end{tabular}

Fuente: Elaboración propia (2021)

\section{Instrumentos}

Cuestionario de cibervictimización (CBV) (Álvarez- García, Dobarro y Núñez, 2014; adaptado por Alvites, 2019). Se evaluaron cuatro dimensiones delciberbullying:cibervictimización verbal escrita, cibervictimización visual, exclusión online y suplantación. La calificación de cada ítem se hizo con una escala tipo Likert de 1 a 4 puntos. La validez factorial del instrumento fue probada por medio de análisis factoriales confirmatorios, lo cual representan de manera adecuada la estructura interna de cuatro factores de la prueba. Con respecto a la validez de criterio se analizaron las puntuaciones del cuestionario del ciberbullying $y$ seis criterios externos evidenciándose una correlación de manera estadísticamente significativa. La confiabilidad del instrumento en Perú, fue testado por Alvites (2019) donde presentó índice de alpha de Cronbach de 0,874. Asimismo, en el presente estudio la confiabilidad fue probada presentando coeficiente de alfa de Cronbach de 0.89 .

Inventario de depresión de Beck II- (DBIII) (Beck, Steer y Brown (1996), adaptado por Espinoza 2016). Evaluó la depresión a través de tres dimensiones: cognitiva, física- conductual y afectiva- emocional. La calificación se realizó a través de una escala Tipo Likert de cuatro alternativas de respuesta. La validez predictiva como instrumento de diagnóstico de cribado ha sido recientemente estudiada en nuestro país en una amplia muestra de población general de entre 18 a 64 años de edad, con buen rendimiento de sensibilidad 100\%, especificidad 99\%, valor predictivo positivo $.72 \mathrm{y}$ valor predictivo negativo 18 (punto de corte $>13$ ). Espinoza (2016) en su estudio realizado en la población universitaria, evaluó la validez de constructo de BDI-II a través de análisis factorial hallando tres factores que explican el 39\% de la varianza total. Finalmente, los índices psicométricos del Inventario de depresión de Beck II- (DBI-II) fueron evaluados por los autores de la escala (Beck, Steer y Brown, 1996) reflejando una buena consistencia interna. (Alpha de Cronbach .76.95) y la fiabilidad del test oscila alrededor de $r=0,8$. En el escenario nacional, en Lima Sur, Espinoza (2016), en una muestra de más de 1000 universitarios, reporto en la confiabilidad un coeficiente alpha de Cronbach de 0.86 para el nivel general y coeficientes superiores a .60 
para las tres áreas en esta población que hallan y componen el inventario. En el presente estudio la confiabilidad fue probada mediante una prueba piloto obteniendo un coeficiente de alfa de Cronbach bueno de .88 .

\section{Tipo y Diseño}

La presente investigación se considera de tipo básico, de manera que está orientada a la búsqueda de nuevos contextos y campos de estudio que posibiliten la obtención de información, la cual refleja características de la realidad, del mismo modo que contribuyan con la actualización y enriquecimiento del entendimiento científico (Hernández et al., 2014).

Se considera al enfoque de tipo cuantitativo, en el sentido que hará uso de la recolección de información en el proceso de comprobación de las hipótesis planteadas, teniendo como base la medición numérica y el análisis a través de una serie de procedimientos estadísticos, los cuales permiten determinar patrones comportamentales y comprobar las teorías que sustentan las variables de interés (Hernández et al., 2014). Así mismo, el alcance del estudio fue explicativo, ya que pretendió establecer las causas de la variabilidad de los niveles de depresión con base en la experiencia de ciberbullying.

La metodología usada se clasifica dentro de la categoría hipotética deductiva que tiene como propósito estudiar y comprender los fenómenos, explicar su origen o los factores que lo generan (Popper, 2008). Por su parte, el diseño fue no experimental - transversal, de manera que se observaron las variables tal y como se presentan en su ambiente natural sin manipulación alguna y en un solo momento determinado (Hernández et al., 2014).

\section{Procedimiento}

Se procedió a solicitar el permiso respectivo a las instituciones universitarias, luego de ello, se diseñó un formulario para recabar la información de forma virtual. Los datos obtenidos se almacenaron mediante el aplicativo de Google
Form. Cabe mencionar, que el formulario presentó de manera clara y concisa información relevante sobre la investigación, además del consentimiento informado, en el que se informa de la participación anónima y el tratamiento confidencial de los datos proporcionados.

\section{Confidencialidad o consentimiento informado}

El presente estudio se desarrolló de acuerdo a los lineamientos éticos propuestos por la Universidad César Vallejo, además de ello se cumplió los principios éticos estipulado en el artículo 25 del código de ética "Trabajo de investigación” de Psicólogos del Perú (1995). La investigación tuvo aspectos formales, tal como el consentimiento informado de cada sujeto, el cual detalló las características del estudio, así como el carácter estrictamente confidencial del mismo. Por otro lado, se respetó el principio de beneficencia, según el cual se busca el bienestar de cada uno de los investigados.

\section{Resultados}

La muestra utilizada en el presente estudio estuvo conformada por 150 participantes, el $81.5 \%$ fueron de sexo femenino $(n=123)$ mientras que el $17.9 \%$ de sexo masculino $(n=27)$. El rango de edad fue entre 16 y 19 años con una media de edad de 18 años y desviación estándar de 0.97. Por otro lado, el 44.1\% $(\mathrm{n}=56)$ de los encuestados se encuentran dentro de la categoría "sin depresión", 32.3\% $(n=41)$ en la categoría "depresión leve", 18.1\% (n = 23) "depresión moderada" y $5.5 \%(\mathrm{n}=7)$ "depresión severa".

La normalidad de las distribuciones de todas las variables usadas en el presente estudio fue evaluada por medio de la prueba de KolmogorovSmirnov. Los resultados evidencian, que ninguna variable presentó distribución normal ( $\mathrm{p}>.05$ ). Como se observa en la Tabla 2, se ejecutó inicialmente un test de correlación entre los puntajes de depresión y los tipos de ciberbullying para un análisis exploratorio de la relación entre las variables investigadas que justifique la posterior ejecución de un análisis de regresión 
lineal múltiple. Se evidencia que la puntuación total de ciberbullying, así como sus cuatro tipos (Cibervictimización verbal - escrita, Cibervictimización Visual, Exclusión online, Suplantación) se correlacionan significativamente con la depresión con coeficientes que oscilan entre $\rho=.37$ y $\rho=.46$. Este análisis previo permitió considerar modelos de regresión, y causalidades en el análisis posterior.

Tabla 2. Resultados del análisis de correlación de Spearman entre las variables ciberbullying y depresión.

\begin{tabular}{cccc}
\hline Variables & Dimensiones & & Depresión \\
\hline & Cibervictimización & $\rho$ & 0.46 \\
verbal - escrita & Sig & .022 \\
& Cibervictimización Visual & $\rho$ & 109 \\
& & 0.44 \\
Ciberbullying & Sig & .000 \\
& Exclusión online & $\mathrm{N}$ & 109 \\
& & $\rho$ & 0.45 \\
& Sig & .000 \\
& & $\mathrm{~N}$ & 109 \\
& & $\rho$ & 0.37 \\
& & Sig & .000 \\
& & $\mathrm{~N}$ & 109 \\
& & $\rho$ & 0.40 \\
& & Siberbullying total & .000 \\
& & $\mathrm{~N}$ & 109 \\
\hline
\end{tabular}

Fuente: Elaboración propia (2021).

Nota: $\rho=$ Coeficiente de correlación de Spearman, Sig = nivel de significancia estadística, $\mathrm{N}=$ número de participantes utilizados en el análisis de correlación.

Considerando los resultados encontrados en el análisis de correlación, se realizó una regresión lineal múltiple para comprender qué dimensiones del ciberbullying podrían considerarse como predictoras de la depresión. Los hallazgos evidenciaron que existe una relación significativa de las dimensiones de ciberbullying cibervictimización verbal - escrita y exclusión online sobre depresión $(\mathrm{F}(2,114)=$ $22,748, \mathrm{p}<.001 ; \mathrm{R} 2$ ajustado $=0.273)$.

La Tabla 3 presenta los coeficientes para todos los predictores significativos. Como se puede observar, la variable que más impactó en los niveles de depresión fue cibervictimización verbal - escrita $(B=0.771, t=3.231, p<.01)$, explicando el $23 \%$ del resultado, mientras que la exclusión $(\mathrm{B}=1.956, \mathrm{t}=2.756, \mathrm{p}<.01)$ tenía un aporte de $8 \%$ de la varianza de la depresión.
Cabe resaltar que los otros tipos de ciberbullying no presentaron impactos significativos en la depresión.

Tabla 3. Regresión lineal múltiple para depresión

\begin{tabular}{cccccc}
\hline Variables del modelo & B & $\begin{array}{c}\text { Error padrón del } \\
\text { coeficiente }\end{array}$ & B & T & $\begin{array}{c}\text { Significancia } \\
\text { estadística }\end{array}$ \\
\hline $\begin{array}{c}\text { Cibervictimización verbal - } \\
\begin{array}{c}\text { escrita } \\
\text { Exclusión online }\end{array}\end{array}$ & 0.771 & 0.239 & 0.321 & 3.231 & $\mathbf{0 . 0 0 2}$ \\
\hline $\mathrm{R}=0.534 ; \mathrm{R}^{2}=0.285 ; \mathrm{R}^{2}$ ajustado $=0.273$ & 0.706 & 0.279 & 2.756 & $\mathbf{0 . 0 0 7}$ \\
\hline
\end{tabular}

Fuente: Elaboración propia (2021).

\section{Discusión y Conclusiones}

Considerando la Teoría Cognitiva de Beck (1987) que postula que el origen de los síntomas depresivos se da por la interpretación que realizan de los eventos y que las experiencias de ciberbullying y soledad están presentes en la cotidianidad y subjetividad de los adolescentes, se planteó como hipótesis general que el ciberbullying impacta en la variable depresión en los estudiantes encuestados. Los resultados a los que se arribaron por medio de los análisis de correlación y regresión demuestran que esta hipótesis se contrasta parcialmente.

En el análisis de correlación se encontró que todos los tipos de ciberbullying se relacionan con la depresión de manera positiva. La relación encontrada entre ciberbullying y depresión está acorde a los resultados de Girgin (2019), Hill et al. (2016), Alvites (2019), Silva Salazar (2020), Dervishi, Lala y Ibrahimi (2019), Arhin et al. (2019), Tajahuercel, Franco y Juárez (2018) y Cañas et al. (2019) quienes también encontraron correlación significativa entre ciberbullying y depresión. También corrobora lo encontrado en un estudio peruano realizado por Arévalo (2020) cuyos resultados revelaron que existe relación positiva, moderada y significativa entre ambas variables.

Los análisis de correlación no proporcionan evidencia de causalidad y los estudios previos realizados, tanto nacionales comointernacionales, usaron solo análisis de correlación, se consideró apropiado realizar un análisis de regresión para encontrar la dimensión de ciberbullying que 
sirve como antecedente de la depresión. Esa hipótesis se basó en el hecho de que los afectados producto del ciberbullying vivencian un conjunto de condiciones emocionales negativas, en donde el sentimiento de angustia emocional alcanza niveles preocupantes (Hinduja y Patchin, 2008; Ybarra y Mitchell, 2004) y que los efectos más usuales propios de quienes han sido expuestos a la cibervictimización consideran frustración, tristeza e ira. Los resultados corroboraron parcialmente la hipótesis general ya que dos tipos de ciberbullying (verbal - escrita y exclusión) se muestran como variables preditoras del modelo de forma significativa explicando el $27 \%$ de la variabilidad de la depresión.

Es innegable que la depresión tiene su origen multicausal el cual integra factores psicosociales, genéticos y biológicos. (Beck y Alford, 2009) por lo que se espera que el modelo propuesto no explique el $100 \%$ de la variabilidad de este constructo. Desde la Teoría Cognitiva, Beck (1991) se postula que los individuos sienten daño emocional por la interpretación que realizan de los eventos y no por cómo éstos se manifiestan en realidad, el vivenciar ataques de ciberbullying tiene repercusiones en el campo emocional de las personas al ser percibidos, clasificados, interpretados y evaluados como algo que puede generar un daño real, que deja la imagen social desvalorizada y que no hay forma de ser enfrentada. Esta percepción de daño real y aumento de la importancia que se le puede dar a un ciberbullying en principales plataformas, puede explicarse con base a que los esquemas cognitivos actuales tienen a las redes sociales como componente fundamental en el desarrollo de cada individuo, de la cual las personas adquieren retroalimentación constante y una gran necesidad de aprobación. Por ello, recibir respuestas negativas de las redes puede generar una percepción errónea de la realidad con ideas de fracaso frente a retos futuros, interpretación negativa de la realidad, y constante autoevaluación desfavorable de sí mismo, característico de un cuadro de depresión (Beck, 1987).

El presente estudio demuestra que la cibervictimización verbal-escrita impacta en la variable depresión en universitarios de Lima. Este tipo de ciberbullying hace referencia a expresiones verbales de connotación violenta difundidas a través de TIC como teléfono, móvil o Internet, lo que viene a ser el tipo de ciberbullying más común, debido a la normalización de lenguaje violento en los comentarios realizados en redes sociales, eso justifica el impacto encontrado en los niveles de depresión en adolescentes, considerando el valor que se le agrega a recibir refuerzo positivos en los post que realizan y el impacto emocional que puede acarrear el tener el efecto opuesto al deseado. Por otro lado, la exclusión online también se presentó como variable predictora ya que al actuar hace que la persona se aísle de su círculo social en el cual interactúa, utilizando estrategias que incluyen la exclusión y rechazo tales como el bloqueo y eliminación en la lista de amigos o grupos. Aliándose de métodos como ignorar a la persona, bloquearla, eliminarla de la lista de amigos o grupos.

Los otros tipos de ciberbullying como son el visual y la suplantación no impactaron en la variable depresión. Para explicar este hecho se deja como hipótesis la falta de información de personas que experimentaron ciberbullying en categorías moderado (4.3\%) y grave (ningún participante se clasificó en esta categoría) ya que la muestra presentó, casi por completo, niveles de ciberbullying leve (95.7\%). En el caso de los encuestados se encuentran dentro de la categoría de haber sido víctimas de mínimos ataques de ciberbullying y uno reporta haber sido víctima de ataques de ciberbullying en nivel medio y ningún participante calificó en niveles altos de ciberbullying. Serán necesarios nuevos estudios que abarquen muestras más homogéneas en función al sexo y que reciban información de personas con experiencia de ciberbullying más severas, para poder corroborar o refutar lo encontrado en el presente estudio sobre que tipos de ciberbullying no son antecedentes de depresión en adolescentes.

Se concluye que el hallazgo de mayor relevancia encontrado en el presente estudio, es que explora no solo la relación entre ciberbullying y depresión, sino que evalúa esa relación según 
los diferentes tipos de ciberbullying, así como determinar qué componentes sirven como predictores de aumento o disminución de los niveles de depresión en adolescentes.

Las limitaciones del presente trabajo están relacionadas a las restricciones propias de las características de la muestra y a las características de realizar un estudio transversal. La ausencia de información que pueda robustecer la discusión de algunos campos como la evaluación de relaciones familiares o sociales limita la propuesta de hipótesis explicativas para los resultados encontrados. Se espera que las limitaciones de este estudio sean un factor instigador para que se desarrollen nuevas investigaciones hipotetizando relaciones con nuevas variables para así expandir el conocimiento científico en esta área y se estima que más estudios con otros tipos de características sociodemográficas de la población peruana aumentarán y reforzarán el cuerpo de evidencia a este respecto. Finalmente, se espera que datos como estos puedan fomentar estrategias preventivas conscientes de las mayores especificidades de la depresión en estudiantes peruanos.

\section{Referencias Bibliográficas}

Álvarez-García, D., Dobarro, A. \& Núñez, J. (2014). Validez y fiabilidad del cuestionario de cibervictimización en estudiantes de secundaria. Aula Abierta, 43, 32-38. https://doi.org/10.1016/j. aula.2014.11.001

Alvites-Huamaní, C. (2019). Adolescencia, ciberbullying y depresión, riesgos en un mundo globalizado. Eitc@net, Revista científica electrónica de Educación y Comunicación en la Sociedad del Conocimiento, 19 (1), 210-234. https://doi.org/10.30827/eticanet.v19i1.11867

Alvites-Huamaní, C. (2020). COVID-19: Pandemia que impacta en los estados del ánimo. CienciAmérica, 9(2), 1-10. Número Especial. https://doi.org/10.33210/ca.v9i2.327

Arévalo Cárdenas, L. S. (2020), Ciberbullying y depresión en estudiantes de secundaria de instituciones educativas públicas del Rímac, 2020. (Tesis de pregrado). Universidad César
Vallejo, Lima, Perú. https://doi.org/10.1186/ s13104-019-4300-6

Arhin, D. K., Asante, K. O., Kugbey, N., \& OtiBoadi, M. (2019). The relationship between psychological distress and bullying victimisation among school-going adolescents in Ghana: a cross-sectional study. BMC Res Notes, 12 (1), 1-5.

Beck, A. \& Alford, B. (2009). Depresión: causas y tratamiento. 2da edición Philadelphia: Universidad de Pennsylvania.

Beck, A. (1991). Terapia Cognitiva de la Depresión. Bilbao: Biblioteca de Psicología

Beck, A. T. (1987). Cognitive models of depression. Journal of Cognitive Pychotherapy, 1, 5-37.

Beck, A. T., Steer, R. A. \& Brown, G. K. (1996). BDI-II. Beck Depression Inventory-second edition. Manual. San Antonio: The Psychological Corporation. https://doi.org/10.1037/t00742000

Brewer, G. \& Kerslake, J. (2015). Cyberbullying, self-esteem, empathy and loneliness. Computers in Human Behavior, 48, 255-260. https://doi. org/10.1016/j.chb.2015.01.073

Cañas, E., Estévez. E., Marzo, J., \& Piqueras, J. (2019) Ajuste psicológico en cibervíctimas y ciberagresores en educación secundaria. Anales de Psicología, 35 (3). https://doi.org/10.6018/ analesps.35.3.323151

Craig, G. (2001). Desarrollo Psicológico. México: Pearson Educación. http://www.uade. inpsiquiatria.edu.mx/tesis/tesis_alma_garcia.pdf Dervishi, E., Lala, M. \& Ibrahimi, S. (2019). School bullying and symptoms of depression. Archives of Psychiatry and Psychotherapy (2), 48-55. https://doi.org/10.12740/APP/103658

Domínguez, J., \& Portela, I. (2020). Violencia a través de las TIC: comportamientos diferenciados por género. RIED. Revista Iberoamericana de Educación a Distancia, 23(1), 273-286. https:// doi.org/10.5944/ried.23.2.25916

Espinoza, A. (2016). Estandarización y propiedades psicométricas del inventario de depresión de Beck (BDI-II) en estudiantes de una universidad privada de Lima Sur (Tesis de pregrado). Universidad Autónoma del Perú, 
Lima, Perú.

Garaigordobil, M. (2015). Ciberbullying en adolescentesyjóvenes del País Vasco: Cambioscon la edad. Anales de Psicología, 31(3), 1069-1076. https://doi.org/10.6018/analesps.31.3.179151

Garaigordobil, M., \& Oñederra, J. A. (2010). La violencia entre iguales. Revisión teórica y estrategias de intervención. Madrid, España: Pirámide

Giménez, A. M. (2015). Ciberbullying: análisis de su incidencia entre estudiantes y percepciones del profesorado. Murcia: Universidad de Murcia. https://www.tdx.cat/handle/10803/312158

Girgin, C. (2019). Forgiveness and cyberbullyıng in Turkısh adolescents. Academic Journals, 14 (14), 512-517. https://doi.org/10.5897/ ERR2019.3786

Hernández, R., Fernández, C., \& Baptista, P. (2014). Metodología de la investigación. (6aed.). McGraw-Hill. https://bit.ly/3rHghMj

Hill, R. M., Mellick, W., Temple, J. R., \& Sharp, C. (2016). The role of bullying in depressive symptoms from adolescence to emerging adulthood: A growth mixture model. Journal of Affective Disorders, 207, 1-8. https://doi. org/10.1016/j.jad.2016.09.007

Hinduja, S., \& Patchin, J. W. (2010). Bullying, cyberbullying, and suicide. Archives of suicide research, 14(3), 206-221. https://doi.org/10.1080 /13811118.2010.494133

Hoff, D. L., \& Mitchell, S. N. (2009). Cyberbullying: Causes, effects, and remedies. Journal of Educational Administration, 47, 652-655. https:// doi.org/10.1108/09578230910981107

Kim, S. (2018). Cyberbullying Victimization and Adolescent Mental Health: Evidence of Differential Effects by Sex and Mental Health Problem Type. Journal of Youth and Adolescence, 47(3), 661-672. https://doi.org/10.1007/s10964017-0678-4

Li, Q. (2008). A cross-cultural comparison of adolescents' experience related to cyberbullying. Educational Research, 50(3), 223-234. https:// doi.org/10.1080/00131880802309333

Matamala Riquelme, C. (2016). Uso de las TIC en el hogar: Entre el entretenimiento y el aprendizaje informal, 2016, de la Comisión Nacional de
Investigación Científica y Tecnológica. https:// doi.org/10.4067/S0718-07052016000400016 OMS. (2017). La OMS pone foco en la depresión, segunda causa de muerte entre los jóvenes. Organización Mundial de la Salud. https://www. who.int/topics/depression/es/\#: :text=La\%20 depresi \% C $3 \%$ B 3 n \% 20 es $\% 20$ un $\% 20$ trastorno,cansancio $\% 20 \mathrm{y} \% 20 \mathrm{falta} \% 20 \mathrm{de} \% 20$ concentraci $\% \mathrm{C} 3 \% \mathrm{~B} 3 \mathrm{n}$

OMS. (2017). Mientras la depresión encabeza la lista de causas de enfermedad. Organización Mundial de la Salud. https://www3.paho.org/ $\mathrm{hq} /$ index.php? option $=$ com_content $\& v i e \mathrm{w}=$ article\&id=13102: depression-lets-talk-sayswho-as-depression-tops-list-of-causes-of-illhealth\&Itemid $=1926 \&$ lang $=$ es

OMS. (2021). Depresión. https://www.who.int/ es/news-room/fact-sheets/detail/depression

Ortega-Barón, J., Begoña, I., Carrascosa, L., Clemente, A., (2019). Satisfacción con la vida, sintomatología depresiva, y soledad en víctimas de ciberbullying. International Journal of Developmental and Educational Psychology INFAD Revista de Psicología, 4(1), 355362. https://doi.org/10.17060/ijodaep.2019. n1.v5.1609

Ortega-Barón, J., Buelga, S., \& Cava, M. J. (2016). The influence of school climate and family climate among adolescents victims of cyberbullying. Comunicar, 24(46), 57-65. https:// doi.org/10.3916/C46-2016-06

Polaino-Lorente, A. (1995). Los Trastornos Afectivos en la Infancia. Las depresiones infantiles. En: Rodríguez Sacristán (ed.) Psicopatología del niño y del adolescente, Tomo I, (pp. 655-688). Universidad de Sevilla.

Popper, K. (2008). La lógica de la investigación científica. Madrid: Tecnos

Rumiche, M., \& Solís, B. (2021). Los efectos positivos y negativos en el uso de las Tecnologías de la Información y Comunicación en educación. Hamut' ay, 8 (1), 27-36. https://doi.org/10.21503/ hamu.v8i1.2233

Renés-Arellano, P., Hernández-Serrano, M-J., Caldeiro-Pedreira, M-C. y Alvites-Huamaní, C. (2021). Contravalores del ethos digital percibidos por futuros formadores. Comunicar, 62 (26), 57 - 
67. https://doi.org/10.3916/C69-2021-05

Sandoval, R., \& Caballero, J. (2016). Asociación entre bullying-ciberbullying y conducta suicida en adolescentes de colegios públicos y privados de la ciudad de Piura. http://200.62.226.189/ PuebloContinente/article/view/1270/1100 Silva Salazar, G. (2020). Depresión y ansiedad asociada al ciberbullying en estudiantes de medicina de la Facultad de Ciencias Médicas de la Universidad Central del Ecuador, noviembre 2018 - mayo 2019 (Tesis de posgrado). Universidad Central del Ecuador, Quito, Ecuador. http:// www.dspace.uce.edu.ec/handle/25000/20859

Smith, P. K. (2006). Ciberacoso: naturaleza y extensión de un nuevo tipo de acoso dentro y fuera de la escuela. Paper presentado al Congreso Educación Palma de Mallorca.

Stopcyberbullying. (s.f.). ¿Qué es el ciberacoso?. https://www.stopbullying.gov/cyberbullying/ what-is-it

Tabares, A. S. G., Núñez, C., Osorio, M. P. A., \& Aguirre, A. M. G. (2020). Riesgo e Ideación Suicida y su Relación con la Impulsividad y la Depresión en Adolescentes Escolares. Revista Iberoamericana de Diagnóstico y Evaluación-e Avaliação Psicológica, 1(54), 147-163. https:// doi.org/10.21865/RIDEP54.1.12

Tajahuerce, I., Franco, G., \& Juárez, J (2018): Ciberbullying y género: nuevos referentes en la ocupación de los espacios virtuales. Estudios sobre el Mensaje Periodístico 24 (2), 1845-1859. https://doi.org/10.5209/ESMP.62250

Walrave, M., \&Walrave, H. (2009). Cyberbullying: Predicting Victimisation and Perpetration. Children \& Society, 25, 59-72. https://doi. org/10.1111/j.1099-0860.2009.00260.x

Willard, N. E. (2006). Cyberbullying and Cyberthreats: Responding to the challenge of online social cruelty, threats and distress. Eugene, Oregon: Center for Safe and Responsible Internet Use.

Ybarra, M. L., \& Mitchell, K. J. (2004). Online aggressor/targets, aggressors, and targets: a comparison of associated youth characteristics. Journal of Child Psychology and Psychiatry, 45(7), 1308-1316. https://doi.org/10.1111/j.14697610.2004.00328.x 


\section{Anexos}

\section{CUESTIONARIO DE CIBERVICTIMIZACIÓN (CBV) \\ Hoja de Respuestas}

Apellidos y Nombres:

Edad:

Grado:

Sección:

Indicaciones: A continuación, encontrarás una lista de oraciones, lee con atención el contenido de cada pregunta y marca con un aspa $(X)$ una de las cuatro alternativas de respuesta. Recuerda que no hay respuestas buenas o malas, por eso lo importante es que respondas sinceramente.

\begin{tabular}{|c|c|c|c|c|c|}
\hline $\mathrm{N}^{0}$ & PREGUNTAS & NUNCA & $\begin{array}{l}\text { POCAS } \\
\text { VECES }\end{array}$ & $\begin{array}{l}\text { MUCHAS } \\
\text { VECES }\end{array}$ & SIEMPRE \\
\hline 1 & $\begin{array}{l}\text { Se han hecho pasar por mí en Internet publicando comentarios a mi nombre, como } \\
\text { si fuese yo. }\end{array}$ & & & & \\
\hline 2 & $\begin{array}{l}\text { Han copiado conversaciones privadas mías y se las han enviado a otros, para } \\
\text { dañarme }\end{array}$ & & & & \\
\hline 3 & $\begin{array}{l}\text { Alguna persona no me ha admitido o me ha expulsado de su equipo en juegos } \\
\text { online, sin haber hecho yo nada malo que lo justifique }\end{array}$ & & & & \\
\hline 4 & $\begin{array}{l}\text { Han colgado en Internet fotos mías trucadas (modificadas), para hacerme daño } 0 \\
\text { reírse de mí. }\end{array}$ & & & & \\
\hline 5 & $\begin{array}{l}\text { Alguien se ha hecho pasar por otra persona, para reírse de mí a través de Internet } \\
\text { o del teléfono móvil }\end{array}$ & & & & \\
\hline 6 & $\begin{array}{l}\text { Me han echado o no me han aceptado en la lista de contactos de algún chat, red } \\
\text { social (por ej., Tuenti) o programa de mensajería instantánea (por ej., Messenger, } \\
\text { WhatsApp), sin haber hecho nada, solo por ser yo }\end{array}$ & & & & \\
\hline 7 & Me han enviado fotos o vídeos «fuertes», desagradables para mí. & & & & \\
\hline 8 & He recibido llamadas a mi móvil, que no contestan, supongo que para fastidiar. & & & & \\
\hline 9 & $\begin{array}{l}\text { Han colgado en Internet fotos o vídeos reales comprometidos, sin mi permiso, para } \\
\text { hacerme daño o reírse de mí. }\end{array}$ & & & & \\
\hline 10 & $\begin{array}{l}\text { Se han publicado en Internet informaciones que había dado en secreto, para que } \\
\text { no se lo dijesen a nadie, y que me dañan }\end{array}$ & & & & \\
\hline 11 & He recibido llamadas para insultarme o burlarse de mí. & & & & \\
\hline 12 & $\begin{array}{l}\text { Me han bloqueado el acceso al correo electrónico, a una red social (Tuenti) o a un } \\
\text { programa de mensajería instantánea (Messenger, WhatsApp), cambiando mi } \\
\text { contraseña }\end{array}$ & & & & \\
\hline 13 & $\begin{array}{l}\text { Se han burlado de mí con comentarios ofensivos } 0 \text { insultantes en las redes } \\
\text { sociales }\end{array}$ & & & & \\
\hline 14 & Me han pegado, lo han grabado y luego lo han difundido & & & & \\
\hline 15 & $\begin{array}{l}\text { He recibido insultos a través de mensajes cortos de texto (sms) o programas de } \\
\text { mensajería instantánea (por ej., WhatsApp). }\end{array}$ & & & & \\
\hline 16 & $\begin{array}{l}\text { Se han hecho pasar por mí en Twitter, Facebook, Tuenti..., creando un falso perfil } \\
\text { de usuario (foto, datos personales) con el que se me ha insultado o ridiculizado }\end{array}$ & & & & \\
\hline 17 & $\begin{array}{l}\text { He recibido mensajes anónimos (sms, WhatsApp), en los que se me amenazaba o } \\
\text { atemorizaba }\end{array}$ & & & & \\
\hline 18 & $\begin{array}{l}\text { Se han hecho quejas falsas sobre mí en algún foro, red social o juego online, que } \\
\text { han hecho que me expulsasen }\end{array}$ & & & & \\
\hline 19 & $\begin{array}{l}\text { Han reenviado a otras personas, para dañarme, correos o mensajes privados que } \\
\text { yo había enviado }\end{array}$ & & & & \\
\hline 20 & $\begin{array}{l}\text { Me han obligado a hacer algo humillante, lo han grabado y luego lo han difundido } \\
\text { para burlarse de mí }\end{array}$ & & & & \\
\hline 21 & $\begin{array}{l}\text { Me han amenazado públicamente, a través de las redes sociales (Tuenti, Twitter, } \\
\text { Facebook. . .) }\end{array}$ & & & & \\
\hline 22 & Se ponen de acuerdo para hacerme el vacío (ignorarme) en las redes sociales & & & & \\
\hline 23 & He recibido llamadas anónimas, para amenazarme 0 atemorizarme & & & & \\
\hline 24 & He recibido comentarios sexuales no deseados a través de Internet & & & & \\
\hline 25 & $\begin{array}{l}\text { Alguien que ha conseguido mi contraseña ha enviado mensajes molestos a algún } \\
\text { conocido, como si hubiese sido yo, para meterme en líos }\end{array}$ & & & & \\
\hline 26 & Se han publicado rumores falsos sobre mí en alguna red social & & & & \\
\hline
\end{tabular}




\section{$\underline{\text { Inventario de Depresión de Beck (BDI-2) }}$}

Nombre:

Estado

Civil.

Edad:

Sexo.

Ocupación

Educación: Fecha:

Instrucciones: Este cuestionario consta de 21 grupos de afirmaciones. Por favor, lea con atención cada uno de ellos cuidadosamente. Luego elija uno de cada grupo, el que mejor describa el modo como se ha sentido las últimas dos semanas, incluyendo el día de hoy. Marque con un círculo el número correspondiente al enunciado elegido Si varios enunciados de un mismo grupo le parecen igualmente apropiados, marque el número más alto. Verifique que no haya elegido más de uno por grupo, incluyendo el ítem 16 (cambios en los hábitos de Sueño) y el ítem 18 (cambios en el apetito)

\section{Tristeza}

$0 \quad$ No me siento triste.

$1 \quad$ Me siento triste gran parte del tiempo

2 Me siento triste todo el tiempo.

3 Me siento tan triste o soy tan infeliz que no puedo soportarlo.

\section{Pesimismo}

$0 \quad$ No estoy desalentado respecto del mi futuro.

1 Me siento más desalentado respecto de mi futuro que lo que solía estarlo.

$2 \quad$ No espero que las cosas funcionen para mí.

3 Siento que no hay esperanza para mi futuro y que sólo puede empeorar.

\section{Fracaso}

$0 \quad$ No me siento como un fracasado.

1 He fracasado más de lo que hubiera debido.

2 Cuando miro hacia atrás, veo muchos fracasos.

3 Siento que como persona soy un fracaso total.

\section{Pérdida de Placer}

$0 \quad$ Obtengo tanto placer como siempre por las cosas de las que disfruto.

$1 \quad$ No disfruto tanto de las cosas como solía hacerlo.

2 Obtengo muy poco placer de las cosas que solía disfrutar.

$3 \quad$ No puedo obtener ningún placer de las cosas de las que solía disfrutar.

\section{Sentimientos de Culpa}

$0 \quad$ No me siento particularmente culpable.

Me siento culpable respecto de varias cosas que he hecho o que debería haber hecho. Me siento bastante culpable la mayor parte del tiempo.

3 Me siento culpable todo el tiempo.

\section{Sentimientos de Castigo}

$0 \quad$ No siento que este siendo castigado

1 Siento que tal vez pueda ser castigado.

2 Espero ser castigado.

3 Siento que estoy siendo castigado.

7. Disconformidad con uno mismo.

$0 \quad$ Siento acerca de mi lo mismo que siempre.

1 He perdido la confianza en mí mismo.

2 Estoy decepcionado conmigo mismo.

3 No me gusto a mí mismo. 


\section{Autocrítica}

$0 \quad$ No me critico ni me culpo más de lo habitual

1 Estoy más crítico conmigo mismo de lo que solía estarlo

2 Me critico a mí mismo por todos mis errores

3 Me culpo a mí mismo por todo lo malo que sucede.

9. Pensamientos o Deseos Suicidas

$0 \quad$ No tengo ningún pensamiento de matarme.

1 He tenido pensamientos de matarme, pero no lo haría

2 Querría matarme

3 Me mataría si tuviera la oportunidad de hacerlo.

\section{Llanto}

$0 \quad$ No lloro más de lo que solía hacerlo.

$1 \quad$ Lloro más de lo que solía hacerlo

$2 \quad$ Lloro por cualquier pequeñez.

3 Siento ganas de llorar, pero no puedo.

\section{Agitación}

$0 \quad$ No estoy más inquieto o tenso que lo habitual.

1 Me siento más inquieto o tenso que lo habitual.

2 Estoy tan inquieto o agitado que me es difícil quedarme quieto

3 Estoy tan inquieto o agitado que tengo que estar siempre en movimiento o haciendo algo.

\section{Pérdida de Interés}

$0 \quad$ No he perdido el interés en otras actividades o personas.

1 Estoy menos interesado que antes en otras personas o cosas.

2 He perdido casi todo el interés en otras personas o cosas.

3 Me es difícil interesarme por algo.

\section{Indecisión}

0 Tomo mis propias decisiones tan bien como siempre.

1 Me resulta más difícil que de costumbre tomar decisiones

$2 \quad$ Encuentro mucha más dificultad que antes para tomar decisiones.

3 Tengo problemas para tomar cualquier decisión.

14. Desvalorización

$0 \quad$ No siento que yo no sea valioso

1 No me considero a mi mismo tan valioso y útil como solía considerarme

2 Me siento menos valioso cuando me comparo con otros.

3 Siento que no valgo nada.

15. Pérdida de Energía

0 Tengo tanta energía como siempre.

1 Tengo menos energía que la que solía tener.

$2 \quad$ No tengo suficiente energía para hacer demasiado

3 No tengo energía suficiente para hacer nada.

16. Cambios en los Hábitos de Sueño

$0 \quad$ No he experimentado ningún cambio en mis hábitos de sueño.

$1^{\text {a }}$. Duermo un poco más que lo habitual.

1b. Duermo un poco menos que lo habitual.

2a Duermo mucho más que lo habitual.

2b. Duermo mucho menos que lo habitual 
$3^{a}$. Duermo la mayor parte del día

3b. Me despierto 1-2 horas más temprano y no puedo volver a dormirme.

\section{Irritabilidad}

$0 \quad$ No estoy tan irritable que lo habitual.

1 Estoy más irritable que lo habitual.

2 Estoy mucho más irritable que lo habitual.

3 Estoy irritable todo el tiempo.

\section{Cambios en el Apetito}

$0 \quad$ No he experimentado ningún cambio en mi apetito.

$1^{\text {a. }} \quad$ Mi apetito es un poco menor que lo habitual.

1b. Mi apetito es un poco mayor que lo habitual.

2a. Mi apetito es mucho menor que antes.

2b. Mi apetito es mucho mayor que lo habitual

$3^{a}$. No tengo apetito en absoluto.

3b. Quiero comer todo el día.

19. Dificultad de Concentración

$0 \quad$ Puedo concentrarme tan bien como siempre.

$1 \quad$ No puedo concentrarme tan bien como habitualmente

2 Me es difícil mantener la mente en algo por mucho tiempo.

3 Encuentro que no puedo concentrarme en nada.

\section{Cansancio o Fatiga}

$0 \quad$ No estoy más cansado o fatigado que lo habitual.

1 Me fatigo o me canso más fácilmente que lo habitual.

2 Estoy demasiado fatigado o cansado para hacer muchas de las cosas que solía hacer.

3 Estoy demasiado fatigado o cansado para hacer la mayoría de las cosas que solía

\section{Pérdida de Interés en el Sexo}

$0 \quad$ No he notado ningún cambio reciente en mi interés por el sexo.

1 Estoy menos interesado en el sexo de lo que solía estarlo.

2 Estoy mucho menos interesado en el sexo.

3 He perdido completamente el interés en el sexo. 